\title{
Phytoseiidae (Acari: Mesostigmata) from the Atlantic Forest in Rio de Janeiro, Brazil, with complementary description of Amblyseius impeltatus Denmark \& Muma
}

\author{
Ana Cristina Cerqueira Cavalcante ${ }^{1}$; Peterson Rodrigo Demite ${ }^{2}$; Antônio Carlos Lofego ${ }^{3}$ \& Fabio Akashi Hernandes ${ }^{4}$ \\ 1 Universidade Federal do Amazonas (UFAM), Instituto de Ciências Exatas e Tecnologia (ICET). Itacoatiara, AM, Brasil. \\ ORCID: http://orcid.org/0000-0002-1023-8579. E-mail: anacris.cavalcante@gmail.com \\ 2 Universidade Federal de Mato Grosso (UFMT), Instituto de Biociências (IB), Centro de Biodiversidade, Programa de Pós-Graduação em Zoologia \\ (PPGZ00). Cuiabá, MT, Brasil. ORCID: http://orcid.org/0000-0001-6376-5463. E-mail: peterson_demite@yahoo.com.br (corresponding author) \\ 3 Universidade Estadual Paulista "Júlio de Mesquita Filho" (UNESP), Instituto de Biociências, Letras e Ciências Exatas (IBILCE), \\ Departamento de Ciências Biológicas. São José do Rio Preto, SP, Brasil. ORCID: http://orcid.org/0000-0002-3495-6858. E-mail: ac.lofego@unesp.br \\ ${ }^{4}$ Universidade Federal de Santa Catarina (UFSC), Departamento de Ecologia e Zoologia (ECZ). Florianópolis, SC, Brasil. \\ ORCID: http://orcid.org/0000-0003-3504-2609. E-mail: abakashi@gmail.com
}

\begin{abstract}
Phytoseiid mites (Acari: Phytoseiidae) have been largely used for the biological control of arthropod pests. However, information on the phytoseiid fauna associated with plants of the Rio de Janeiro State, Brazil is still scanty. Surveys were carried out in two areas of Atlantic Forest in Rio de Janeiro State - Jaguanum island and Paraty. We report 11 species of seven genera from all three subfamilies. A complementary re-description is provided for Amblyseius impeltatus Denmark \& Muma, 1973 and measurements are provided for ten other species.
\end{abstract}

Keywords. Coast; Island; Predator; Survey; Parasitiformes.

\section{INTRODUCTION}

The Atlantic Forest occupies mainly the East coast of Brazil, but also parts of Argentina and Paraguay. In Brazil, it covers more than 1 million $\mathrm{km}^{2}$ (MMA, 2015). Due to the high degree of endemism and the exceptional loss of original habitat, the Atlantic Forest is considered a hotspot and a world priority conservation area (Myers et al., 2000; Rezende et al., 2018). About 900 mite (Acari) species have been registered in this biome, belonging to 124 families of both Acariformes and Parasitiformes (Giupponi et al., 2017).

Phytoseiid mites (Parasitifomes: Phytoseiidae) are used as biological control agents in several regions of the world (Gerson et al., 2003; McMurtry et al., 2013; Knapp et al., 2018). Therefore, it is one of the most studied mite families, with more than 2,700 described species worldwide; in Brazil, about 220 species have been reported (Demite et al., 2014, 2021).

More than 150 phytoseiid species have been recorded from the Atlantic Forest (Giupponi et al., 2017), which represents about $60 \%$ of the species of this family registered in Brazil. However, several areas of this biome have been poorly or not yet investigated. The majority of species was reported from São Paulo state, where most samplings have been conducted (e.g., Karg, 1983; Feres \& Moraes, 1998; Gondim-Jr. \& Moraes, 2001; Zacarias \& Moraes, 2002; Feres et al., 2005, 2007; Buosi et al., 2006; Castro \& Moraes, 2007, 2010; Demite et al., 2011; Moraes et al., 2013). Only two studies reported phytoseiids from natural areas of Rio de Janeiro state, both from the same municipality Itatiaia (El-Banhawy, 1984; Kreiter \& Tixier, 2010), and a third study focused on mites of Carica papaya L. (Caricaceae) orchards (Collier et al., 2004).

The aim of this study was to survey the phytoseiid fauna associated with some native and cultivated plants from Rio de Janeiro state.

\section{MATERIAL AND METHODS}

This study was conducted in two areas of the Atlantic Forest in the state of Rio de Janeiro, Brazil: Jaguanum Island $\left(22^{\circ} 59^{\prime} 28^{\prime \prime} \mathrm{S}, 43^{\circ} 55^{\prime} 25^{\prime \prime} \mathrm{W}\right)$ and Paraty $\left(23^{\circ} 13^{\prime} 30^{\prime \prime} S, 44^{\circ} 46^{\prime} 35^{\prime \prime} \mathrm{W}\right)$. In each area, native and cultivated plants were sampled, in April 
of 2007 (Jaguanum Island) and July of 2008 (Paraty). Specimens were mounted in Hoyer's medium; under "Material examined", we list the locality, specimens collected, host plant and collection date. The illustrations were accomplished using a drawing tube attached to an optic microscope equipped with phase contrast (Leica DM 2500). All measurements are given in micrometres $(\mu \mathrm{m})$; each measurement corresponds to the mean for the stated number of individuals, followed by the respective range (min-max) in parentheses. The setal nomenclature adopted were those of Lindquist \& Evans (1965) and Lindquist (1994), as adapted for Phytoseiidae by Rowell et al. (1978) for the dorsum; and by Chant \& Yoshida-Shaul (1991) for the venter. Numbers of teeth on the fixed and movable cheliceral digits do not include the respective apical teeth.

Voucher specimens were deposited in the Acari Collection of Departamento de Ciências Biológicas (DZSJRP-Acari), Universidade Estadual Paulista (UNESP), São José do Rio Preto, São Paulo, Brazil (http://splink.cria. org.br). Holotype micrographs of Amblyseius impeltatus deposited at the collection of Florida Department of Agriculture and Consumer Service, Gainesville, FL, USA, were also examined.

\section{RESULTS AND DISCUSSION}

Eleven phytoseiid species belonging to seven genera from three subfamilies were found. A complementary redescription for Amblyseius impeltatus Denmark \& Muma, 1973 is provided. Morphological information for the other species is given in Table 1.

\section{Family Phytoseiidae Berlese}

\section{Subfamily Amblyseiinae Muma}

\section{Amblyseius impeltatus Denmark \& Muma (Figs. 1-8)}

Amblyseius impeltatus Denmark \& Muma, 1973: 241; Moraes et al., 1986: 16; 2004: 30; Chant \& McMurtry, 2004: 199; 2007: 78.

Amblyseius (Multiseius) impeltatus - Denmark \& Muma, 1989: 91.

Material examined: Paraty: 8 females and 2 males on Cupania sp. (Sapindaceae), 27-VIII-2008.

\section{Female $(n=5)$}

Dorsum (Fig. 1): Dorsal shield smooth, with 6 pairs gland pores ( $g d 1, g d 2, g d 4, g d 5, g d 8$ and $g d 9)$ and 12 pairs of lyrifissures (id1, id2, id4, idx, idm2, idm3, idm4, idm6, is 1, $i d / 2$, idl3 and idl4) visible, 388 (380-395) long and 250 (237-270) wide; with 17 pairs of setae on dorsal shield; setae $r 3$ and $R 1$ inserted in the unsclerotized cuticle. Setae j1 20 (19-20), j3 22 (22-23), j4 9 (8-10), j5 9 (7-10), j6 11 (9-12), J2 14 (14-15), J5 8 (7-9), z2 15 (13-16), z4 11 (10-12), z5 12 (10-14), Z1 13 (12-14), Z4 37 (34-41), Z5 134 (120-140), s4 41 (39-43), S2 14 (13-15), S4 13 (12-14), S5 13 (11-14), r3 12 (11-13), R1 13 (12-14). All dorsal setae smooth, except $Z 5$ serrate.

Peritreme: Extending forward to level of $j 1$.

Venter (Fig. 2): Sternal shield smooth, except for some lateral striation in the anterior region; with three pairs of setae (st 1, st 2 and st 3 ) and two pairs of lyrifissures (iv1 and iv2); distances between st1-st3 53 (52-55), st2-st2 70 (68-73). Seta st4 and lyrifissure iv3 on metasternal plate. Genital shield smooth, st5-st5 84 (82-87). With two pairs of metapodal plate. Ventrianal shield pentagonal, smooth; 97 (95-99) long, 85 (82-89) wide at level of ZV2 and 77 (72-80) wide at anus level; with three pairs of preanal setae (JV1, JV2 and ZV2) and a pair of spheric pores (gv3) posteromesad to JV2. Four pairs of opisthogastric setae on unsclerotized cuticle (JV4, JV5, ZV1 and ZV3). JV5 61 (59-67). All ventral setae smooth.

Spermatheca (Figs. 3 and 8): Calyx bell-shaped 7 (7-9) long. Atrium indistinct, only noticeable by the presence of a ring surrounding embolus (lips) and minor duct.

Chelicera (Fig. 4): Movable cheliceral digit 27 (26-27) long, with three teeth; fixed cheliceral digit 27 (26-28) long, with 8-10 teeth.

Legs (Fig. 5): Leg macrosetae smooth and pointed: Sgel 30 (31-33), Sgell 34 (32-35), Sgelll 43 (42-45), Still 30 (27-31), SgelV 69 (68-71), StilV 52 (51-54), StIV 56 (54-58). Chaetotactic formula of genu II: 2-2/0-2/0-1 and genu III: 1-2/1-2/0-1.

\section{Male $(n=2)$}

Dorsum: Dorsal shield smooth; 270 long and 215 wide. Setae $j 1$ 13, j3 30, j4 3, j5 5-7, j6 5-6, J2 10-11, J5 6-7, z2 11-13, z4 10, z5 10, Z1 10, Z4 33-34, Z5 115-119, s4 36-37, $S 212$, S4 10-11, S5 7-8, r3 11-13, R1 11. Setae $r 3$ and $R 1$ on dorsal shield. All dorsal setae smooth, except $Z 5$ serrate.

Peritreme: Extending forward to the level of $j 1$.

Venter (Fig. 6): Sternogenital shield smooth; ventrianal shield subtriangular, with striation anteriorly to pores (gv3); 104 long, 142-145 wide at anterior corners. JV5 40-44. All ventral setae smooth.

Spermatodactyl (Fig. 7): Shaft of spermatodactyl 20-22 long.

Legs: Leg macrosetae smooth: Sgel 28-29, Sgell 28-29, Sgelll 31-32, Stilll 26-27, SgelV 47-48, StilV 42, StIV 47-49. Chaetotactic formula of genu II and genu III as female.

Remarks: The cervix of the spermatheca of $A$. impeltatus was originally depicted in a narrow and elongated shape, 
Table 1. Measures (mean and range) of eight species of Phytoseiidae of two areas of Atlantic Forest in Rio de Janeiro State (Jaguanum island and Paraty), Brazil.

\begin{tabular}{|c|c|c|c|c|c|c|c|c|c|c|}
\hline \multirow[b]{2}{*}{ Character } & \multicolumn{7}{|c|}{ Amblyseiinae } & \multicolumn{2}{|c|}{ Phytoseiinae } & \multirow{2}{*}{$\begin{array}{c}\text { Typhlodrominae } \\
\text { Leonseius } \\
\text { regularis } \\
\wp(\mathrm{n}=3)\end{array}$} \\
\hline & $\begin{array}{c}\text { Amblyseius } \\
\text { Iargoensis } \\
\bigcirc(n=2)\end{array}$ & $\begin{array}{c}\text { Amblyseius } \\
\text { operculatus } \\
\text { ㅇ }(n=5)\end{array}$ & $\begin{array}{c}\text { Euseius } \\
\text { alatus } \\
\stackrel{Q}{(n=3)}\end{array}$ & $\begin{array}{c}\text { Euseius } \\
\text { mesembrinus } \\
\stackrel{(n=5)}{ }\end{array}$ & $\begin{array}{l}\text { Iphiseiodes } \\
\text { zuluagai } \\
\wp(n=1)\end{array}$ & $\begin{array}{c}\text { Proprioseiopsis } \\
\text { dominigos } \\
\text { 을 }(n=1)\end{array}$ & $\begin{array}{l}\text { Typhlodromips } \\
\text { cananeiensis } \\
\text { O }(\mathrm{n}=1)\end{array}$ & $\begin{array}{c}\text { Phytoseius } \\
\text { marumbus } \\
\stackrel{(n=1)}{ }\end{array}$ & $\begin{array}{c}\text { Phytoseius } \\
\text { woodburyi } \\
\text { 을 }(n=5)\end{array}$ & \\
\hline DSL & $350-387$ & 409 (385-425) & $304(295-312)$ & 315 & 350 & 505 & 320 & 280 & $300(295-305)$ & $378(365-388)$ \\
\hline DSW & $232-235$ & $286(262-300)$ & 225 & $206(200-212)$ & 315 & 380 & 210 & 155 & 155 (150-157) & $280(275-288)$ \\
\hline j1 & $35-41$ & $41(40-43)$ & $28(25-30)$ & $29(29-30)$ & 32 & 25 & 20 & 20 & $32(31-34)$ & $30(29-30)$ \\
\hline j3 & $51-52$ & $58(55-61)$ & $20(20-21)$ & $19(17-21)$ & 35 & 112 & 20 & 24 & $39(37-42)$ & $42(41-45)$ \\
\hline$j 4$ & 7 & $10(9-11)$ & 15 & $9(8-10)$ & 3 & 4 & 18 & 13 & $5(4-6)$ & 5 \\
\hline$j 5$ & 7 & $7(6-7)$ & $15(14-15)$ & $9(9-10)$ & 3 & 3 & 11 & 7 & $6(5-6)$ & 5 \\
\hline j6 & 8 & $10(8-11)$ & $17(14-18)$ & $10(10-11)$ & 3 & 2 & 14 & 7 & $7(6-7)$ & 5 \\
\hline$J 2$ & $10-13$ & $11(10-12)$ & $18(16-19)$ & 12 (11-13), & 3 & - & 14 & 7 & - & $7(6-7)$ \\
\hline 15 & 10 & $9(8-10)$ & $8(7-9)$ & $6(5-6)$ & 4 & 5 & 9 & 7 & $7(7-8)$ & $7(6-8)$ \\
\hline$z 2$ & $10-13$ & $16(13-17)$ & 17 (17-18) & $14(13-15)$ & 2 & 16 & 17 & 7 & $15(14-17)$ & $5(4-6)$ \\
\hline 23 & - & - & - & - & - & - & - & 43 & $36(34-38)$ & $11(10-11)$ \\
\hline$z 4$ & $9-10$ & $15(12-17)$ & $16(16-17)$ & $15(14-16)$ & 2 & 40 & 16 & 6 & $15(14-16)$ & $7(6-7)$ \\
\hline 25 & 7 & $8(7-9)$ & $15(15-16)$ & $9(9-10)$ & 2 & 4 & 14 & 10 & $6(5-6)$ & $5(4-5)$ \\
\hline 21 & $8-14$ & $12(11-12)$ & $18(17-19)$ & $12(12-13)$ & 3 & 6 & 17 & - & - & - \\
\hline 24 & $95-100$ & 139 (132-152) & $18(18-19)$ & 12 & 4 & 150 & 28 & 53 & $97(92-100)$ & $95(88-100)$ \\
\hline 25 & $262-300$ & $297(285-307)$ & $57(55-60)$ & $57(54-59)$ & 117 & 145 & 69 & 68 & $84(81-90)$ & $301(292-315)$ \\
\hline$s 4$ & $92-102$ & $111(104-116)$ & $22(21-23)$ & $22(20-23)$ & 116 & 137 & 20 & 55 & $129(121-140)$ & $90(85-96)$ \\
\hline s6 & - & - & - & - & - & - & - & 65 & $89(87-90)$ & $8(7-8)$ \\
\hline$S 2$ & $12-19$ & $14(12-15)$ & $20(19-21)$ & $15(14-16)$ & 3 & 6 & 20 & - & - & - \\
\hline 54 & $12-13$ & $14(12-16)$ & $20(20-21)$ & $17(16-19)$ & 4 & 6 & 17 & - & - & - \\
\hline S5 & $12-13$ & $14(12-16)$ & $24(21-26)$ & $18(17-20)$ & 5 & 4 & 17 & - & - & $6(6-7)$ \\
\hline$r 3$ & $14-16$ & $16(15-17)$ & $15(15-16)$ & 16 & 7 & 10 & 15 & 42 & $48(45-51)$ & $10(10-11)$ \\
\hline$R 1$ & $12-14$ & $15(11-19)$ & $15(14-15)$ & $13(12-13)$ & 6 & lost/broken & 15 & 15 & - & $10(9-10)$ \\
\hline st1-st3 & $65-68$ & $74(70-77)$ & $52(50-53)$ & $56(54-57)$ & 50 & 60 & 58 & 62 & $60(57-65)$ & $63(62-64)$ \\
\hline$s t 2-s t 2$ & $63-67$ & $80(77-85)$ & $69(68-70)$ & $68(67-71)$ & 82 & 77 & 66 & 65 & $62(62-63)$ & $71(69-73)$ \\
\hline$s t 5-s t 5$ & $52-75$ & $77(74-82)$ & 73 & $71(67-75)$ & 119 & 120 & 62 & 55 & $63(62-64)$ & 77 (76-78) \\
\hline VSL & $105-115$ & $139(130-147)$ & $94(92-96)$ & $96(93-101)$ & 120 & 118 & 110 & 86 & $98(95-101)$ & $118(116-119)$ \\
\hline VSWant & $52-53$ & $86(82-89)$ & $55(53-57)$ & $54(50-57)$ & 124 & 135 & 101 & 46 & $40(37-43)$ & $66(64-68)$ \\
\hline VSWpost & $73-71$ & $92(85-97)$ & $66(63-70)$ & $67(65-69)$ & 110 & 125 & 80 & 49 & $46(45-47)$ & $78(73-82)$ \\
\hline$J V 5$ & $68-70$ & $84(80-88)$ & $33(30-35)$ & $31(30-33)$ & 37 & 57 & 48 & 45 & $53(49-56)$ & $62(59-65)$ \\
\hline calyxL & $24-37$ & $8(7-9)$ & $17(16-18)$ & $16(16-17)$ & 6 & 23 & 7 & 10 & $8-10$ & $11(10-12)$ \\
\hline MDL & $27-30$ & $36(34-37)$ & $22(21-23)$ & 23 & 27 & 35 & 28 & 22 & $21(20-22)$ & $25(20-29)$ \\
\hline MD teeth & 3 & $4-5$ & 1 & 1 & 1 & 2 & 2 & 1 & 1 & 3 \\
\hline $\mathrm{FDL}$ & $30-35$ & $38(35-40)$ & $24(24-26)$ & $25(24-25)$ & 32 & 37 & 28 & 22 & $23(22-25)$ & $(24-30)$ \\
\hline FD teeth & 11 & $13-17$ & $6-7$ & 5 & 12 & 4 & 11 & 3 & $3-4$ & 11 \\
\hline Sgel & $32-43$ & $47(39-52)$ & $21(20-22)$ & $23(22-24)$ & 46 & 20 & - & - & - & $51(50-53)$ \\
\hline Sgell & $38-40$ & $46(39-50)$ & $19(18-19)$ & $23(23-24)$ & 33 & 21 & - & - & - & $43(40-50)$ \\
\hline Sgelll & $50-55$ & $40(38-42)$ & $26(25-26)$ & $26(26-27)$ & 45 & 26 & 19 & - & - & $53(50-55)$ \\
\hline Stilll & $38-42$ & $40(38-42)$ & 20 & 23 & 27 & 26 & - & - & - & $35(35-36)$ \\
\hline SgelV & $122-136$ & $130(125-140)$ & $37(36-38)$ & $34(33-37)$ & 85 & 68 & 27 & 22 & - & $128(123-132)$ \\
\hline StilV & $98-104$ & $93(80-103)$ & $25(23-27)$ & $30(27-32)$ & 52 & 48 & 18 & 24 & $51(50-54)$ & $79(76-82)$ \\
\hline StIV & $65-72$ & $84(80-88)$ & $44(43-45)$ & $51(49-54)$ & 35 & 65 & 38 & 25 & $26(25-27)$ & $59(58-60)$ \\
\hline
\end{tabular}

DSL: Dorsal Shield Length; DSW: Dorsal Shield Width; VSL:Ventrianal Shield Long; VSWant:Ventrianal Shield Wide at level of ZV2; VSWpost:Ventrianal Shield widht at anus level; calyxL: calyx of spermatheca length; MDL: Movable Digit Length; FDL: Fixed Digit Length.

not showing a distinct atrium and both major and minor ducts (Denmark \& Muma, 1973, fig. 15). Subsequently, in a revision of genus Amblyseius, the spermatheca was modified to a stockier shape, with both ducts represented, but with a distinct nodular atrium (Denmark \& Muma, 1989, fig. 477). In our specimens, however, the spermatheca had a bell-shaped calyx and an indistinct atrium, indistinguishable from the major duct (Figs. 4, 8). Micrograph examination of the holotype species (Fig. 8) has revealed the shape of spermatheca matches with the structure described above in our specimens, and do not correspond the previous two illustrations. In addition, all setae on leg IV are herein illustrated on genu (7), tibia (6) and basitarsus (4), while some of the ventral setae were not represented in those previous works.

It is also noteworthy mentioning that despite both papers (Denmark \& Muma, 1973, 1989) stated the holotype is deposited at ESALQ Collection in Piracicaba, Brazil, it is actually deposited at the collection of Florida Department of Agriculture and Consumer Service, Gainesville, FL, USA. 


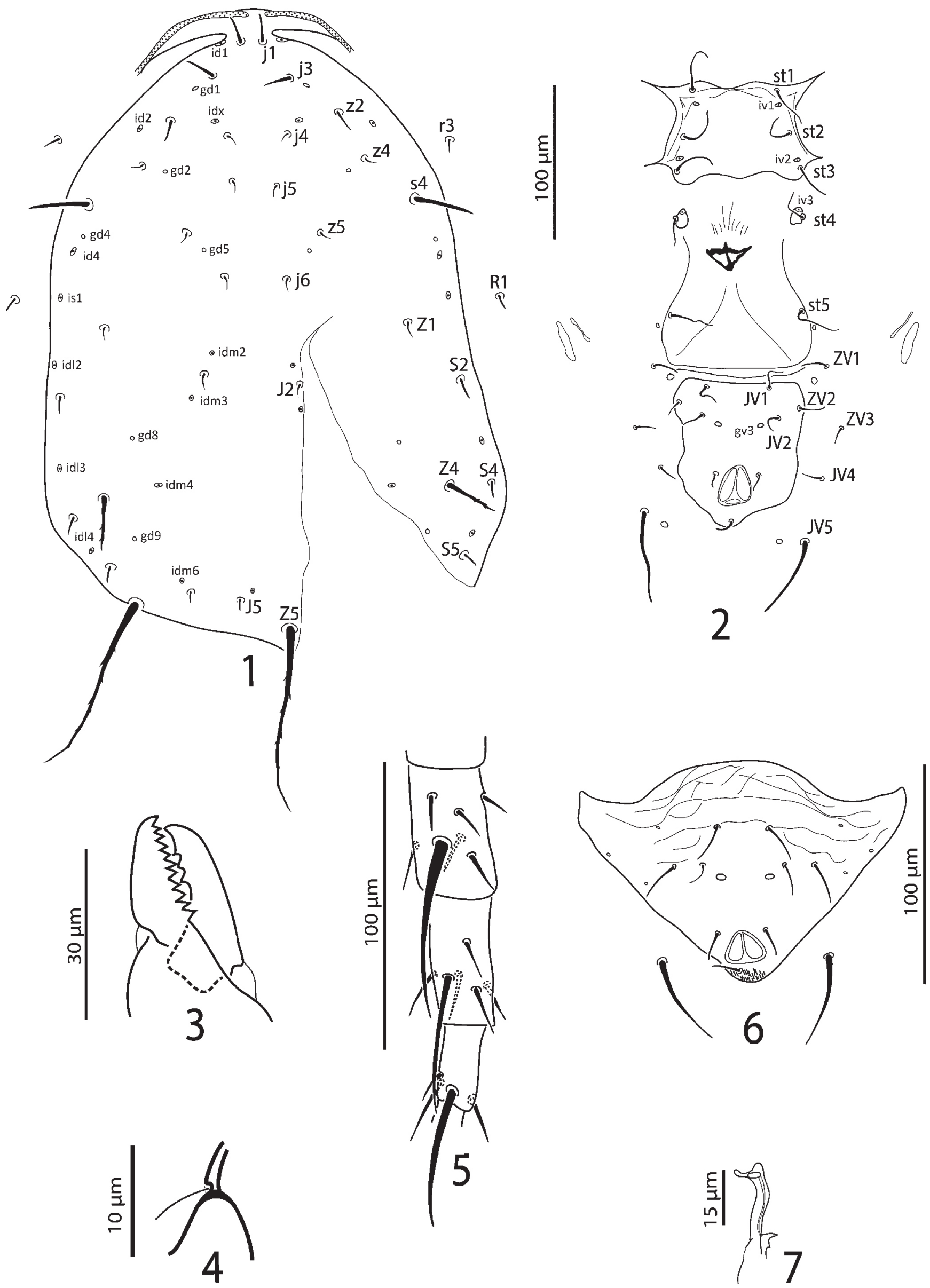

Figures 1-7. Amblyseius impeltatus Denmark \& Muma, 1973. Female (1-5): (1) Dorsal shield; (2) Ventral idiosoma; (3) Chelicera; (4) Spermatheca; (5) Genu, tibia and basitarsus of leg IV. Male (6-7): (6) Ventrianal shield; (7) Spermatodactyl. 


\section{Amblyseius largoensis (Muma)}

Material examined: Jaguanum Island: 2 females on Couroupita guianensis (Lecythidaceae), 20-IV-2007.

\section{Amblyseius operculatus De Leon}

Material examined: Jaguanum Island: 3 females on unidentified plant, 19-IV-2007; 2 females on Persea americana (Lauraceae), 20-IV-2008; 1 female on C. guianensis, 20-IV-2007; Paraty: 5 females on Cupania sp., 27-VII-2008.

\section{Euseius alatus De Leon}

Material examined: Jaguanum Island: 3 females on unidentified plant, 19-IV-2007.

\section{Euseius mesembrinus (Dean)}

Material examined: Jaguanum Island: 13 females and 4 males on unidentified plant, 19-IV-2007, 5 females and 3 males, 20-IV-2007, 5 females and 1 male, 21-IV-2007; 7 females and 3 males on undetermined Leguminosae, 19-IV-2007; 2 females and 1 male on Hibiscus sp. (Malvaceae), 19-IV-2007.

\section{Iphiseiodes zuluagai Denmark \& Muma}

Material examined: Jaguanum Island: 1 female on unidentified plant, 19-IV-2007.

\section{Proprioseiopsis dominigos (El-Banhawy)}

Material examined: Paraty: 1 female on Cupania sp., 27-VII-2008.

\section{Typhlodromips cananeiensis Gondim-Jr. \& Moraes}

Material examined: Paraty: 1 female on Cupania sp., 27-VII-2008.

\section{Subfamily Phytoseiinae Berlese}

\section{Phytoseius marumbus El-Banhawy}

Material examined: Jaguanum Island: 1 female on unidentified plant, 19-IV-2007.

\section{Phytoseius woodburyi De Leon}

Material examined: Jaguanum Island: 2 females on unidentified plant, 19-IV-2007, 11 females and 1 male,

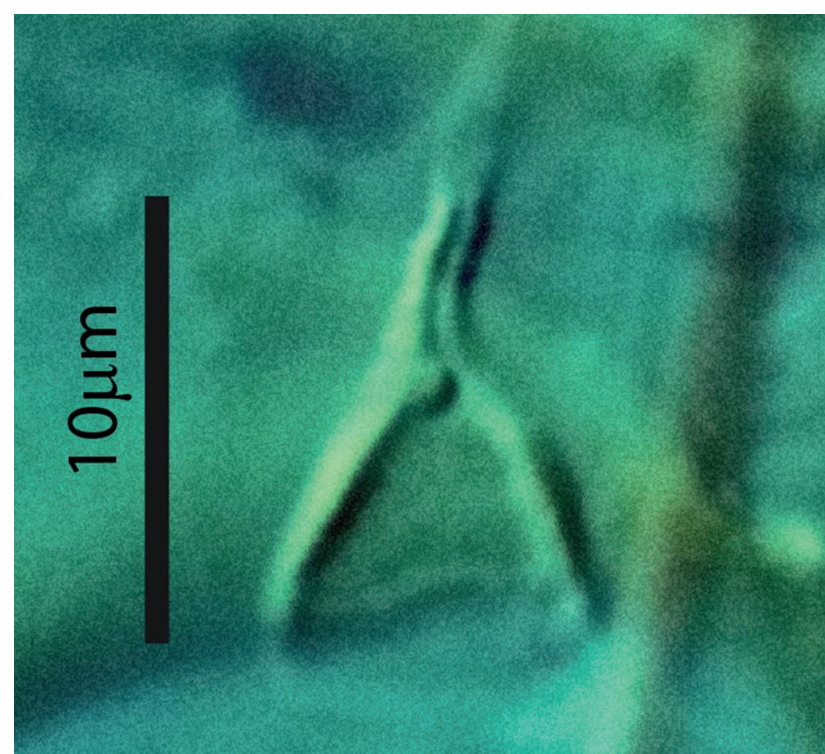

Figure 8. Amblyseius impeltatus Denmark \& Muma. Holotype spermatheca (Photo: Samuel Bolton).

20-IV-2007; 1 female on unidentified Leguminosae, 19-IV-2007.

\section{Subfamily Typhlodrominae Wainstein}

\section{Leonseius regularis (De Leon)}

Material examined: Paraty: 3 females on $P$. americana, 20-IV-2007; 4 females on Cupania sp., 27-VII-2008.

All species found here had previously been reported in the Atlantic Forest in South coast of São Paulo State (Gondim-Jr. \& Moraes, 2001; Moraes et al., 2013; Zacarias \& Moraes, 2002), distant about $400 \mathrm{~km}$ from both areas sampled in this study. This suggests a similarity among phytoseiid mite communities from different areas of Atlantic Forest.

Two species found in this study, T. cananeiensis and P. marumbus, have been found only in the Atlantic Forest so far, suggesting they might be endemic to this biome. Others, like Amblyseius largoensis, Euseius alatus, Iphiseiodes zuluagai, and Proprioseiopsis dominigos, all reported herein, are also frequently registered in several agricultural crops in other localities and biomes in Brazil, indicating phytoseiids from the Atlantic Forest may also migrate to nearby crops and act as natural enemies of pest mites. This reinforces the idea of the native vegetation working as reservoirs of phytoseiids as suggested by previous authors (Tixier et al., 2000; Demite \& Feres, 2005, 2008; Lofego \& Moraes, 2006; Castro \& Moraes, 2007, 2010; Demite et al., 2013; Rezende et al., 2014). Phytoseiids have a major role in ecosystems, regulating phytophagous mite populations in natural environments and agroecosystems (Demite et al., 2013; Lofego $\&$ Moraes, 2006). Surveying natural vegetation areas provides a clearer picture of the real diversity of these organisms, also contributing to understand the structure of mite communities and the species turnover between natural vegetation and adjacent crops. 


\section{ACKNOWLEDGMENTS}

We thank Dr. Samuel J. Bolton (Florida Department of Agriculture and Consumer Services, Division of Plant Industry) for taking and sending pictures of the holotype of Amblyseius impeltatus at the collection of Florida Department of Agriculture and Consumer Service, Gainesville, FL, USA. To Fundação de Amparo à Pesquisa do Estado de São Paulo (FAPESP, Procs. 06/52893-5, 06/57868-9). PRD acknowledges funding from Coordenação de Aperfeiçoamento de Pessoal de Nível Superior (CAPES/PNPD, Proc. 88887.373867/2019-00). $A C L$ and $F A H$ received a research productivity fellowship from the Conselho Nacional de Desenvolvimento Científico e Tecnológico (CNPq, Procs. 310617/2018-9 and 304479/2019-5).

\section{AUTHORS' CONTRIBUTIONS}

Conceived the study: A.C.C.C., P.R.D., A.C.L., F.A.H. Material sampling: P.R.D., A.C.L., F.A.H. Species identification and analysis: A.C.C.C., P.R.D., A.C.L. Illustration: A.C.C.C., P.R.D. Writing, review and editing: A.C.C., P.R.D., A.C.L., F.A.H.

\section{REFERENCES}

Buosi, R.; Feres, R.J.F.; Oliveira, A.R.; Lofego, A.C. \& Hernandes, F.A. 2006. Ácaros plantícolas (Acari) da "Estação Ecológica de Paulo de Faria", estado de São Paulo, Brasil. Biota Neotropica, 6(1): 1-20.

Castro, T.M.M.G. de \& Moraes, G.J. de. 2007. Mite diversity on plants of different families found in the Brazilian Atlantic Forest. Neotropical Entomology, 36(5): 774-782.

Castro, T.M.M.G. de \& Moraes, G.J. de. 2010. Diversity of phytoseiid mites (Acari: Mesostigmata: Phytoseiidae) in the Atlantic Forest of São Paulo. Systematics and Biodiversity, 8(2): 301-307.

Chant, D.A. \& McMurtry, J.A. 2004. A review of the subfamily Amblyseiinae Muma (Acari: Phytoseiidae): Part II. The tribe Amblyseiini Wainstein, subtribe Amblyseiina n. subtribe. International Journal of Acarology, 30(3): 171-228.

Chant, D.A. \& McMurtry, J.A. 2007. Illustrated keys and diagnoses for the genera and subgenera of the Phytoseiidae of the world (Acari: Mesostigmata). West Bloomfield, Indira Publishing House. 219p.

Chant, D.A.\& Yoshida-Shaul, E. 1991. Adult ventral setal patterns in the family Phytoseiidae (Acari: Gamasina). International Journal of Acarology, 17(3): 187-199.

Collier, K.F.S.; de Lima, J.0.G. \& Albuquerque, G.S. 2004. Predacious mites in papaya (Carica papaya L.) orchards: in search of a biological control agent of phytophagous mite pests. Neotropical Entomology, 33(6): 799-803.

Demite, P.R. \& Feres, R.J. 2008. Influência de fragmentos de Cerrado na distribuição de ácaros em seringal. Neotropical Entomology, 37(2): 196-204.

Demite, P.R. \& Feres, R.J.F. 2005. Influência de vegetação vizinha na distribuição de ácaros (Acari) em seringal no município de São José do Rio Preto, São Paulo, Brasil. Neotropical Entomology, 34(5): 829-836.

Demite, P.R.; Lofego, A.C. \& Feres, R.J. 2013. Mite (Acari; Arachnida) diversity of two native plants in fragments of a semideciduous seasonal forest in Brazil. Systematics and Biodiversity, 11(2): 141-148.
Demite, P.R.; Lofego, A.C. \& Feres, R.J.F. 2011. Phytoseiidae (Acari) in forest fragments in the State of São Paulo, Brazil. Zootaxa, 3086: 31-56.

Demite, P.R.; McMurtry, J.A. \& Moraes, G.J. de. 2014. Phytoseiidae Database: a website for taxonomic and distributional information on phytoseiid mites (Acari). Zootaxa, 3795(5): 571-577.

Demite, P.R.; Moraes, G.J. de; McMurtry, J.A.; Denmark, H.A. \& Castilho, R. de C. 2021. Phytoseiidae Database. Available: http://www.lea.esalq.usp.br/ phytoseiidae. Access: 25/04/2021.

Denmark, H.A. \& Muma, M.H. 1973. Phytoseiid mites of Brazil (Acarina: Phytoseiidae). Revista Brasileira de Biologia, 33(2): 235-276.

Denmark, H.A. \& Muma, M.H. 1989. A revision of the genus Amblyseius Berlese, 1914 (Acari: Phytoseiidae). Occasional Papers of the Florida State Collection of Arthropods, 4: 1-149.

El-Banhawy, E.M. 1984. Description of some phytoseiid mites from Brazil (Acarina: Phytoseiidae). Acarologia, 25(2): 125-144.

Feres, R.J.F. \& Moraes, G.J. de. 1998. Phytoseiid mites (Acari: Phytoseiidae) from woody areas in the State of São Paulo, Brazil. Systematic \& Applied Acarology, 3(1): 125-132.

Feres, R.J.F.; Buosi, R.; Daud, R.D. \& Demite, P.R. 2007. Padrões ecológicos da comunidade de ácaros em euforbiáceas de um fragmento de mata Estacional Semidecidual, no Estado de São Paulo. Biota Neotropica, 7(2): 185-194.

Feres, R.J.F.; Lofego, A.C. \& Oliveira, A.R. 2005. Ácaros plantícolas (Acari) da "Estação Ecológica do Noroeste Paulista", estado de São Paulo, Brasil. Biota Neotropica, 5(1): 43-56.

Gerson, U.; Smiley, R.L. \& Ochoa, R. 2003. Mites for pest control. Oxford (UK), Blackwell Science. 539p.

Giupponi, A.P. de L.; Demite, P.R.; Flechtmann, C.H.W.; Hernandes, F.A.; Mendes, A.C.; Migliorini, G.H.; Miranda, G.S. de \& Souza, T.G. 2017. Aracnídeos da Mata Atlântica. In: Monteiro-Filho, E.L. de A. \& Conte, C.E. (Eds.). Revisões em Zoologia: Mata Atlântica. Curitiba, Editora UFPR. p. $129-235$.

Gondim-Jr., M.G.C. \& Moraes, G.J. de. 2001. Phytoseiid mites (Acari: Phytoseiidae) associated with palm trees (Arecaceae) in Brazil. Systematic \& Applied Acarology, 6(1): 65-94.

Karg, W. 1983. Systematische untersuchung der Gattungen und Untergattungen der Raubmilbenfamilie Phytoseiidae Berlese, 1916, mit der beschreibung von 8 neuen Arten. Mitteilungen Zoologisches Museum in Berlin, 59(2): 293-328.

Knapp, M.; Van Hounten, Y.; Van Baal, E. \& Groot, T. 2018. Use of predatory mites in commercial biocontrol: current status and future prospects. Acarologia, 58(Suppl): 72-82.

Kreiter, S. \& Tixier, M.-S. 2010. A new genus and species of phytoseiid mites (Acari: Mesostigmata) from the Brazilian Atlantic Forest. Acarologia, 50(2): 197-205.

Lindquist, E.E. 1994. Some observations on the chaetotaxy of the caudal body region of gamasine mites (Acari: Mesostigmata), with a modified notation for some ventrolateral body setae. Acarologia, 35(4): 323-326.

Lindquist, E.E. \& Evans, G.0. 1965. Taxonomic concepts in the Ascidae, with a modified setal nomenclature for the idiosoma of the Gamasina (Acarina: Mesostigmata). Memoirs of the Entomological Society of Canada, 47: $1-65$.

Lofego, A.C. \& Moraes, G.J. de. 2006. Ácaros (Acari) associados a mirtáceas (Myrtaceae) em áreas de Cerrado no Estado de São Paulo com análise faunística das famílias Phytoseiidae e Tarsonemidae. Neotropical Entomology, 35(6): 731-746.

McMurtry, J.A.; Moraes, G.J. de \& Famah Sourassou, N. 2013. Revision of the lifestyles of phytoseiid mites (Acari: Phytoseiidae) and implications for biological control strategies. Systematic \& Applied Acarology, 18(4): 297-320. 
Ministério do Meio Ambiente (MMA). 2015. Mapa de vegetação nativa na área de aplicação da Lei № 11.428/2006 - Lei da Mata Atlântica (ano base 2009). Brasília, FUNCATE. 84p.

Moraes, G.J. de; Barbosa, M.F. de C. \& Castro, T.M.M.G. de. 2013. Phytoseiidae (Acari: Mesostigmata) from natural ecosystems in the State of São Paulo, Brazil. Zootaxa, 3700(3): 301-347.

Moraes, G.J. de; McMurtry, J.A. \& Denmark, H.A. 1986. A catalog of the mite family Phytoseiidae: references to taxonomy, synonymy, distribution and habitat. Brasília, EMBRAPA-DDT. 353p.

Moraes, G.J. de; McMurtry, J.A.; Denmark, H.A. \& Campus, C.B. 2004. A revised catalog of the mite family Phytoseiidae. Zootaxa, 434: 1-494.

Myers, N.; Mittermeier, R.A.; Mittermeier, C.G.; Fonseca, G.A.B. da \& Kent, J. 2000. Biodiversity hotspots for conservation priorities. Nature, 403(6772): 853-858.

Rezende, C.L.; Scarano, F.R; Assad, E.D.; Joly, C.A.; Metzger, J.P.; Strassburg, B.B.N.; Tabarelli, M.; Fonseca, G.A. \& Mittermeier, R.A. 2018. From hotspot to hopespot: an opportunity for the Brazilian Atlantic Forest. Perspectives in Ecology and Conservation, 16: 208-214.

Rezende, J.M.; Lofego, A.C.; Nuvoloni, F.M. \& Navia, D. 2014. Mites from Cerrado fragments and adjacent soybean crops: does the native vegetation help or harm the plantation? Experimental \& Applied Acarology, 64(4): 501-518.

Rowell, H.J.; Chant, D.A. \& Hansell, R.I.C. 1978. The determination of setal homologies and setal patterns on the dorsal shield in the family Phytoseiidae (Acarina: Mesostigmata). The Canadian Entomologist, 110(8): 859-876.

Tixier, M.-S.; Kreiter, S. \& Auger, P. 2000. Colonization of vineyards by phytoseiid mites: their dispersal patterns in the plot and their fate. Experimental \& Applied Acarology, 24(3): 191-211.

Zacarias, M.S. \& Moraes, G.J. de. 2002. Mite diversity (Arthropoda: Acari) on euphorbiaceous plants in three localities in the State of São Paulo. Biota Neotropica, 2(2): 1-12. 